\title{
Client Experiences of Emergency Assistance Services
}

\author{
Tanya Mackay \\ University of South Australia \\ lan Goodwin-Smith \\ Flinders University
}

\begin{abstract}
More people are requiring emergency assistance on a regular basis, placing a significant burden on service providers. However, there is little literature on client perceptions of emergency assistance, and whether their needs are being met. This study aimed to address this gap by interviewing people who had recently sought emergency assistance in South Australia. Sixteen people (nine women) aged between 27 and 55 participated in this study. Participants reported that they were seeking assistance (mainly food) due to bills, particularly relating to electricity, but also telephone, gas and car registration. Most people had their needs met to some extent, but many said that they had received insufficient or inappropriate food. Recommendations were provided around establishing compulsory service standards and partnerships, and reviewing the types of assistance provided (e.g. providing vouchers and healthy food parcels).
\end{abstract}

Keywords: emergency assistance, emergency relief, social services, wrap around services, social inclusion

The number of people requiring emergency assistance is increasing (Anglicare Australia, 2010; Engels, Nissim \& Landvogt, 2009; Frederick \& Goddard, 2008; Nguyen, 2011), stretching support services beyond their capacity (AnglicareSA, 2014). Despite this growth in demand, there is little literature on the way that emergency assistance recipients perceive various types of emergency assistance, and whether these services meet their needs, particularly in an Australian context. This study aims to explore client experiences of emergency assistance services in South Australia, in an effort to improve client experiences and outcomes.

Emergency relief has been described as the 'safety net to the safety net' (Babbington \& Donato-Hunt, 2007; p.40); a residual form of welfare that community agencies provide to people experiencing a temporary financial crisis (Babbington \& Donato-Hunt, 2007; Landvogt, 2006; Nguyen, 2011). Emergency assistance generally consists of food, gift cards, vouchers, cash, cheques, utility payments, household goods, clothing, transport assistance or pharmacy assistance (Australian Council of Social Services (ACOSS), 1997a, ACOSS 1997b, ACOSS, 1999; Anglicare Australia, 2010; Babbington \& Donato-Hunt, 2007; Engels, Nissim \& Landvogt, 2009). Australian emergency assistance programs are often funded by the Commonwealth Government. However, agencies also receive support through fundraising and donations of goods or money, as well as state and local governments (Anglicare Australia, 2010; Engels, 2006).

Anglicare Victoria (2003) categorises three types of crisis that tend to cause people to seek emergency assistance. These include:

- isolated crises (temporary support due to an unexpected cost)

- periodical crises (assistance required for extended periods of time)

- long term crises (ongoing support due to long term poverty) (Anglicare Victoria, 2003).

Corresponding author: Ian Goodwin-Smith (ian.goodwinsmith@flinders.edu.au) 
Crisis triggers include life events (e.g. injury, unemployment, separation and violence), along with increased living costs and decreased affordability of necessities like housing. Lack of financial management skills, drug and alcohol addictions, and socioeconomic circumstances also contribute to the need for emergency assistance (SACOSS, 2011).

Emergency assistance agencies are becoming an ongoing resource for people who cannot afford basic necessities (Anglicare Australia, 2010; Engels, Nissim \& Landvogt, 2009; Frederick \& Goddard, 2008; Nguyen, 2011). This reliance on emergency assistance to 'make ends meet' has been argued to reflect increasing living costs and inadequate social security payments (ACOSS 2011b; Bath, 1985; Engels, Nissim \& Landvogt, 2009; National Council of Social Services (NCOSS), 1985; Nguyen, 2011). The majority of people seeking emergency assistance support are receiving some form of welfare payment (ACOSS, 1997a, ACOSS 1997b, ACOSS, 1999; ACOSS, 2011a; Babbington \& Donato-Hunt, 2007; Bath, 1985; Engels, Nissim \& Landvogt, 2009; Griffiths, 1975), but these welfare payments aresignificantly below the three most widely accepted poverty measures (the $50 \%$ of median income OECD measure, the $60 \%$ of median income used in the UK and The European Union, and the Henderson poverty line) (ACOSS, 2008; ACOSS, 2011c). Furthermore, recent reports suggest that the number of Australian people living in poverty has increased (ACOSS, 2008). The notion of emergency assistance as a 'once off' band aid solution to temporary crisis is challenged by this data, which potentially foreshadows an ongoing need for emergency assistance agencies to act as links between those experiencing extended periods of financial crisis and other services addressing underlying issues.

Previous studies into emergency assistance within Australia have provided a fairly consistent snapshot of the people who utilise these services. They are primarily female, between 25-49 years in age (ACOSS, 1999), thus falling into as the 'workforce age'. A majority of clients are born in Australia, with Aboriginal and Torres Strait Islander people tending to be overrepresented (ACOSS, 1999; King, Bellamy, Swann, Gavarotto and Coller, 2009). The Australian Council of Social Services (ACOSS) (1999) note that people from non-English speaking backgrounds comprise approximately $13-17 \%$ of the respondents to their national annual emergency relief data collection project. Furthermore, people seeking emergency assistance are generally sole parents or living alone (ACOSS, 2011a).

Studies suggest that a low percentage of people seeking emergency assistance were undertaking paid work at the time (ACOSS, 2011a; Babbington \& Donato-Hunt, 2007; Engels, Nissim \& Landvogt, 2009; Green, Zappala \& Parker, 2000), however, this figure is increasing, with more recipients employed on a casual or part time basis and/or experiencing decreasing hours or wages (Engels, Nissim \& Landvogt, 2009). Emergency assistance recipients are most likely to be housed in private rental dwellings, followed by public housing (ACOSS, 1999; ACOSS, 2011a; Green, Zappala \& Parker, 2000; King et. al. 2009), whilst studies suggest that approximately one quarter of recipients are homeless (ACOSS, 1999; ACOSS, 2011a; Engels, Nissim \& Landvogt, 2009). Although very few emergency recipients are paying off a mortgage, this number has increased (Engels, Nissim \& Landvogt, 2009). The circumstances noted as key reasons for seeking assistance include unemployment, utility bills, accommodation costs, health costs, transport costs, family breakdown, domestic violence/sexual assault and rising living costs, including food (ACOSS, 1997a, ACOSS 1997b, ACOSS, 1999; ACOSS, 2011a; Babbington \& Donato-Hunt, 2007; Engels, Nissim \& Landvogt, 2009; Green, Zappala \& Parker, 2000; King et. al. 2009; Nguyen, 2011). Centrelink's 'breach' process is causing considerable suffering to people and forcing them to seek to emergency assistance from a system already under pressure (Babbington \& DonatoHunt, 2007; Engels, Nissim \& Landvogt, 2009; Engels, 2006).

Engels (2006) explored the provision of emergency assistance in Melbourne (Victoria, Australia) through the lens of the services that provide emergency relief. The services that 
participated in this survey suggested that they were facing an increasing demand for emergency assistance, a diversifying client group, the 'stagnation' of funding and a shrinking volunteer pool (Engels, 2006). Other studies have also expressed concerns with funding issues, with Babbington and Donato-Hunt (2007) suggesting that holes in the broader welfare 'safety net' were placing pressure on an underfunded emergency assistance system. Engels' (2006) study suggested that staffing problems were linked to these funding issues, with participating agencies noting that funding was insufficient to cover the paid staff required to replace a receding number of volunteers. Given that volunteers have traditionally made up a majority of the emergency assistance workforce, this is particularly problematic for providers (ACOSS, 2011b; Engels, 2006). Centrelink payment suspensions were also indicated as a significant concern in regards to pressures on emergency assistance, with providers suggesting that Centrelink was a difficult agency to work with, particularly when it came to referring clients (Engels, 2006).

Gribble (2006) also explored emergency assistance from an organisational perspective in Melbourne, examining the networks between emergency assistance providers, and between providers and broader community services. Gribble (2006) identified limits to networking between agencies, including the high percentage of volunteer staff, lack of resources and differences in service delivery and approach. Gribble (2006) concluded that further development of emergency assistance networks could be beneficial in several ways, including allowing for the coordination of services, the development and sharing of knowledge, and improved access to broader services and increased access to resources.

Despite a significant body of research about the nature of emergency assistance recipients (who they are) and the factors that contribute to their need for assistance, few studies explore client experiences. Frederick and Goddard's (2008) qualitative study on the views of Australian emergency assistance service users found that emergency relief recipients often feel embarrassment, humiliation and shame for needing to ask for emergency assistance. Although these feelings had been noted in previous research, it could be argued that Frederick and Goddard's (2008) qualitative approach added depth and richness to this body of knowledge by seeking to understand what emergency relief means in 'human terms'. Participants also voiced concerns about the limits to help available at emergency assistance sites (Frederick and Goddard, 2008). This could be said to echo service provider concerns about lack of resourcing and inability to meet demand, as noted by Engles (2006). Frederick and Goddard's (2008) study also highlighted positive experiences, emphasising the importance of workers being able to appropriate goods and services, and demonstrate kindness and respect to recipients (Frederick \& Goddard, 2008).

A study undertaken by Babbington and Donato-Hunt (2007), which focused on food insecurity in New South Wales, documented similar findings to Frederick \& Goddard about the experience of emergency assistance recipients. They found that although $80 \%$ of respondents provided positive feedback about their emergency assistance experiences, $20 \%$ discussed unhelpful experiences and inappropriate or inadequate services. King et al. (2009) reiterated the need for compassion and non-judgement in their New South Wales study of client experiences, and the demeaning effects that people can experience when seeking emergency assistance.

As demonstrated above, there have been several studies into the characteristics of people seeking emergency assistance in Australia, and of emergency assistance itself. However, most of this research has been based in two states: Victoria and New South Wales. Furthermore, the national 'People in Financial Crisis' survey undertaken by the Australian Council of Social Services (ACOSS) provides no state-specific data. As such, little is known about those seeking emergency relief in South Australia. This study seeks to explore the South Australian experience of emergency relief, providing a snap shot of the reasons why 
South Australians seek emergency assistance, the types of assistance they seek, and their coping mechanisms for dealing with financial crises. The recommendations developed through this study aim to help emergency assistance services to better meet the needs of emergency assistance clients.

\section{Methodology}

The research employed a qualitative approach in order to focus on meanings and experiences, aiming to understand 'understand people, rather than measure them' (Neuman, 2000; Sarantakos, 1998). The study utilised one-to-one semi-structured interviews in order to gather data in a focused, yet conversational, participant driven manner (Neuman, 2000; Sarantakos, 1998). Each interview took approximately 15 minutes and was held in a private interviewing room located within the emergency assistance site the participant was recruited from. They were conducted in private quiet rooms at four emergency assistance sites. The interviews used a combination of closed and open-ended questions regarding experiences with emergency assistance services to elicit both specific and more in depth, conversational data. The study was clearly explained to each of the participants and each person was provided an information sheet outlining the study, prior to signing a consent form. Participants were asked for permission to audio tape the interviews. One person declined to be recorded, so in depth notes were undertaken by the researcher. The interview questions focused on eliciting the experiences and stories of participants, based on a review of relevant literature.

\section{Participants}

Participants were recruited from clients presenting for emergency assistance at a South Australian non-government organisation. The sites were based in northern outer metropolitan, southern outer metropolitan and inner city locations. Sixteen people participated in the study, of whom nine were female and seven were male. They were aged between 27 and 55 years of age, with the average age of participants being 39 . Of the 16 participants, four lived alone, six lived with partners and children, and four were a single living with children. Two people were living with children and grandchildren. All participants had received emergency assistance within South Australia.

\section{Data Analysis}

The data was analysed using thematic analysis, as described by Braun and Clarke (2006). The first step in this process was transcribing the audio recordings and incorporating hand written notes taken during the interviews. The first author then read through each data set and made notes on stand out points. This provided an initial list of ideas that then formed the basis for coding the data. Coding was undertaken manually and involved the identification of interesting or similar extracts, repeated patterns, and accounts that resisted or contradicted dominant narratives. These codes were then analysed in a broader context and grouped into themes that were reviewed with relation to previous research exploring emergency assistance.

\section{Ethics}

Ethical approval for this research was sought and granted. To ensure voluntary participation, the offer to participate occurred following the provision of emergency assistance services, so that clients did not feel participation was mandatory in order to receive emergency assistance. This is consistent with methods previously used by Engels, Nissim and Landvogt (2009) in a large scale study on the cost of living, financial pressure and emergency relief in Victoria. Clients were informed that participation was voluntary, that they could withdraw at any time and that participation or withdrawal would not affect their access to services. To ensure informed consent, participants were provided with an information sheet, which was verbally outlined by the researcher. Additionally, all participants were required to sign a 
consent form, which was read to them prior to signing to ensure they understood the conditions of participating in the study.

\section{Findings}

Reasons for seeking assistance

Participants in the study overwhelmingly reported that they were seeking emergency assistance due to bills, primarily related to electricity. Telephone (both mobile and landline), gas and car registration costs were also reported to be causing participants financial difficulty. Participants suggested that the costs associated with these bills are increasing and that they were often all due around the same time causing further strain on their budgets. Several participants also noted costs associated with accommodation, particularly the cost of relocating. Three participants stated that costs such as medication, transport and medical treatments for health problems experienced by themselves or a family member were causing financial difficulty. Costs associated with children were also noted to be a contributing factor to needing to seek emergency assistance. These costs included requiring more food due to school holidays or children being home from school, children's medical bills, clothing, costs associated with education for children such as excursions and, as described by participant Eight, wanting to ensure children 'don't miss out'. Only one participant reported selfeducation expenses to be causing financial strain. Two participants reported substance abuse as their reason for needing emergency assistance, and one participant stated job loss and associated debt as the cause of their financial difficulties.

All sixteen participants had sought food from emergency assistance centres, with four specifically stating they had sought vouchers to buy food with. Two people reported that they had also sought help with bills, one person was seeking clothing and hygiene products due to relocation related cost pressures, and one person stated that they had sought assistance with medical prescriptions.

\section{Client needs and satisfaction}

A majority of participants described the assistance provided as having met their need at that time. However, two people suggested that the service provided was unsuitable and therefore did not meet their needs. Both these participants spoke about feeling unheard and feeling perceived as undeserving of assistance. As Participant Four said,

Well I sit here now and I see lovely blankets out there and I was only given a thin blanket like that so I really felt hurt that my need wasn't seen. I felt as if I already had everything, that I was just coming for extra assistance. I had a letter there to state what I needed but it was if I wasn't heard.

Despite the majority of participants describing their needs as having been met, they also expressed concerns about not receiving enough suitable food, and about the suitability of the food provided. Participants generally felt that the food provided by emergency assistance sites often wasn't enough to feed large families and families with teenage children. Although the limitations of emergency assistance sites were acknowledged, participants explained that children, particularly teenagers, eat a lot and that food parcels were often insufficient to feed the family until they next received an income.. As stated by Participant Two,

I don't think it's enough at times ... they say ... that's for a family of three. It's just not enough. It's like for one person, sort of, you know what I mean? You know my son's like a big eater. That would be a meal for him so to speak, so I think they need to look at their food amounts.

Although participants were thankful for the items received, in particular for free bread provided at various sites, six people mentioned an inability to create complete meals with the non-perishable items often available at emergency assistance centres. Not being able to utilise these items for school lunches and the inappropriateness of food for health conditions was also noted. As Participant 12 said, 
A lot of their stuff is things you've already got in your cupboard you don't really need, more need fresh milk vegetables and stuff like that for the kids for school and things. Some of the agencies don't realise that they are more of a priority need than tinned fruit and spaghetti and stuff because that's no good to give kids for school.

One participant noted that since being diagnosed with a heart condition, buying the 'right food' cost him more, and that a lot of the food at the centre was 'bad' for his heart, limiting his choices.. However it was evident that the four people who spoke of having dietary requirements in this study were unable to get suitable food at emergency assistance sites. At two of the interview sites, participants were provided gift cards to supermarkets instead of a food parcel in line with those sites polices. All eleven participants from these sites stated that what they were given met their need; while fourteen of the overall sixteen participants stated that they preferred a voucher over a food parcel. Two participants stated that vouchers allowed them to get food that met either a dietary requirement or medical need, whilst one suggested that he could get 'better quality food' using a voucher. One woman said that, with a voucher, she could get things like nappies, which were not always available at the emergency assistance centre. It was suggested by two participants that vouchers could be a lower amount, combined with food items, as this would give them the ability to get fresh food such as meat but also have the staples required to make a complete a meal. The participants in this study described vouchers as giving them an opportunity to participate in decision making. Participant 11 expressed this:

I think it's better because in a food parcel they give you weird things like pineapple or you know like random things that you can't eat as a staple meal, whereas if you have the card you can go get a piece of bread, a piece of meat, a piece of cheese and you know that can be a sandwich where pineapple and tuna sort of don't go together.

All sixteen participants in the study spoke of having positive experiences at emergency assistance centres. These positive experiences included people who worked at emergency assistance sites being respectful, friendly, helpful and considerate, which made the participants feel comfortable and welcome. Several participants also described polite, understanding and non-judgemental workers as a positive. Participant One said, "No one's being rude to me. I've always been treated with respect and understanding and you don't get that everywhere. Obviously being non-judgmental is part of it and the volunteers are quite friendly, which I think is good". Participant Five said that, Everyone else has been really helpful so I actually feel really comfortable coming here and really appreciated". Participant Eight noted, "Well you've always got a friendly face and all that kind of stuff. The staff are always nice and they don't look down on you".

\section{Being heard - personalised responses to individual situations}

Personalised responses to individual situations were important to the people who participated this study. Acts such as a hug, asking about children or health and listening to and talking about issues that may be affecting the client were described as being positive experiences that made participants feel more comfortable and accepted at emergency assistance centres. One participant stated that a hug from a worker made her feel like she was looked at 'as a human being, not an object'. Another participant expressed that the workers often made her feel better about seeking emergency assistance, as she felt down about needing the service. Two participants reported that their most positive experiences stemmed from interactions with emergency assistance workers who had used the service themselves, or who had experienced similar socio-economic stresses. They felt these workers understood their story and situation. Participant 12 said, "Very friendly staff - always they try and help you if you've got a problem they'll sit down and talk to you about it and ... they really seem to care about your wellbeing and ... like, this morning, I got asked how my knee was and what was I having done to my knee". Participant 14 noted the importance of 
the interpersonal contact at emergency assistance sites, "Just compassion the people give you here they try and help you out the best they can, and they don't judge".

In this study, reports of positive experiences of emergency assistance appeared more prevalent then negative accounts, with only five participants reporting experiences that were unsupportive or upsetting. These experiences primarily revolved around feeling unheard or judged. Not being heard, or an inability of workers to see need, resulted in participants feeling hurt. Two participants described feeling as though they were perceived as being in their situation because of a fault or because of their own choices, with one expressing that those kind of negative experiences can be 'crushing'. One participant said that she felt as though she was seen as not being in need, whilst two participants felt that if they complained about the service, they were seen as being 'crazy' or 'difficult'.

Participant Five commented on the centrality of the interpersonal dynamic with staff and volunteers:

Basically, it's all based on the way you are treated, because a lot of the time the assumption is that people are in the situation through their own volition on their own design, when that may not actually be the case and they may actually be doing an amazing job when they've had all their resources stripped away from them, and some places are just incredibly rude. I've had really, really inappropriate things said to me about, well how can you afford lipstick.

\section{Discussion}

The perspectives of those who seek emergency assistance, such as those highlighted in this study, are an important tool in ensuring that the development of polices and service in the area of emergency assistance reflect and fit client need. That the findings of this study so closely echo those of Frederick and Goddard's (2008) Victorian study is significant, and further amplifies the voices and needs of those who use and are affected by emergency assistance services. However, in contrast, while many participants in Frederick and Goddard's (2008) study reported some positive experiences, in this study they exceeded the number of negative accounts. Some differences were observed, however, in relation to what service users individually identified as positives or negatives of their experience.

The reasons for seeking emergency assistance reflect findings in previous studies, where utility bills, accommodation costs, health costs, unemployment, and rising living costs have been identified as the primary causes as to why people seek relief (ACOSS, 1999; ACOSS, 2011a; Babbington \& Donato-Hunt, 2007; Engels, Nissim \& Landvogt, 2009; Green, Zappala \& Parker, 2000). However, unlike with previous studies, costs associated with raising children were clearly causing financial pressure for the participants of this study, who expressed a desire for children to not be seen as 'lacking', and who articulated feelings of guilt for not being able to provide. This is consistent with previous research in which food was identified as the primary need of emergency assistance applicants, as available money is often used to pay bills or rent, leaving them a shortfall for food (ACOSS, 1999; Engels, Nissim \& Landvogt, 2009).

Clients in this study were primarily seeking food, due to financial pressure caused by utility bills, accommodation and costs associated with children. They were engaged with multiple services and emergency assistance providers, and a majority had sought emergency assistance in the previous year. The negative experiences of participants as described in this and previous studies are concerning (Babbington \& Donato-Hunt, 2007; Frederick \& Goddard, 2008; Nguyen, 2011). The applicants of emergency assistance are often experiencing a range of complex issues, being marginalised and impacted by a range of effects associated with low socioeconomic status (Liddell, 2000). To feel their worthiness for service is questioned, or to be treated in a rude or demeaning manner can only be said to 
further compound these issues. This process of disempowerment, and feelings of being judged, as evident in participant accounts, seems to illustrate that the notions of 'deserving or undeserving poor' still sometimes permeate emergency assistance services. Comparatively positive experiences, which were much more commonly reported in this study, have the potential to develop a sense of dignity, empowerment and capacity in clients. Being heard, feeling respected and receiving empathic and honest responses from workers was shown to minimise feelings of embarrassment, to make participants 'feel better' and to build a sense of comfort. These types of behaviours from workers were also reported as 'helpful' in Frederick and Goddard's (2008) study of emergency relief experiences, and this finding reinforces the impact that workers can have on clients seeking emergency assistance

It was evident throughout this study that participants felt vouchers and the choice associated with these, also contributed to a sense of empowerment, and to practical and suitable outcomes. Vouchers have been identified as having several advantages, with the most commonly cited being that they provide clients with choice (King et. al., 2009). This was reflected in participant descriptions of assistance, with vouchers being said to give them choice and to empower them to make their own decisions. However, food parcels were not disregarded. Rather, a combination of both seemed to reflect participant need, allowing them to get fresh food, whilst also giving them 'staples' to make complete meals. Furthermore, although the concept of food parcels is often constructed in the literature as being essentially disempowering (Mitchell, 2004; Poppendieck, 1998), this was not clearly evident in this study: participants were appreciative of receiving food parcels, and saw value in them. Instead, participants reported unsuitable food items or insufficient amounts of food as unhelpful or degrading, leading to feelings that their needs weren't heard. This suggests that evaluation of - and changes to - food parcels to better reflect clients' needs may positively impact clients' sense of worth and self-esteem. It is notable that storing and maintaining the fresh food participants desired in food parcels is a challenge for emergency assistance sites, however utilising a combination of voucher and food parcel as advocated by those interviewed in this study may mitigate this issue. These concerns echo those identified in Frederick and Goddard's (2008) study, and highlight the limits that people face when needing assistance. The ACOSS Emergency Relief Handbook (2011a) identifies that one of the disadvantages of providing food parcels is that it can be difficult to cater to individual requirements. This was highlighted in the current study, where it was evident that the food available was not always suitable for the diverse range of clients that present at emergency assistance sites. In relation to those with health or dietary requirements, the ACOSS Emergency Relief Handbook states that, 'it is important for them to have easy access to good quality healthy foods when food relief is required, as this will build health and may ultimately lessen the demand for ER in the long-term' (ACOSS, 2011a, p.138)

Participants' positive descriptions of 'personalised touches', of being heard and being able to talk about their underlying issues, reflect a need for the emergency assistance experience to be more than a quick, band-aid provision of goods if it is to be a positive experience, This reflects the accounts of participants in Frederick and Goddard's study, who suggested that 'a personalised service would be helpful' (2008, p.278). however, current resourcing and funding limits this possibility. FaHCSIA (2012) guidelines around emergency assistance encourage partnerships between emergency assistance and services that address a range of needs, such as mental health, violence, addiction, and people newly arrived in communities (ACOSS, 2011a). Networking may be one way in which more holistic services that explore the complex factors affecting clients' lives could be developed without placing increasing demand on providers (Barker, 2004; Gribble, 2006; Nguyen, 2011). This kind of holistic, listening and nurturing approach to emergency assistance not only has merit in terms of producing sustainable social and personal results which address complex needs, it has the capacity to make receiving assistance positive and empowering at the most basic human level - at the level of being listened to and cared about, rather than being dealt with 
expediently. Further development of emergency assistance as a cross-referring link between clients and resources will contribute to empowering clients through enhancing their sense of being listened to, whilst further empowering through building capacity and addressing underlying issues exasperating financial distress and need for emergency assistance in the first place.

However highlighting the stories of those utilising emergency assistance, this study has further strengthened existing arguments for increased funding to the sector, for networking of services, and for an examination of the types of assistance provided through a lens of client dignity.

Implications for practice Implication1: Establish compulsory standards for emergency assistance providers to promote a culture or respect, empathy and compassion

Although ACOSS provide a handbook for emergency assistance providers, it is not a compulsory standard. This study recommends and affirms the logic of Frederick and Goddard's (2008) proposal for service standards for agencies involved in the provision of emergency assistance and Smith's (2004) and Wilson's (2006) proposal for a peak body to monitor such standards. It is evident that participants' experiences in this study varied at different emergency assistance sites and, as such, a system with more consistency around empowering practice appears appropriate. It has been shown that participants' experiences may be heavily dependent on how they are treated by emergency assistance workers. Consistent standards that promote a culture of respect, empathy and compassion amongst workers could only serve to reduce negative and degrading experiences and replicate some of the highly positive experiences expressed by participants in this study (Liddell, 2000; Nguyen, 2011; Smith, 2004; Wilson 2006).

\section{Recommendation 2: Establish partnerships to address the underlying issues contributing to financial distress}

This study also recommends active engagement with the FaHCSIA (2012) guidelines, which advocate for partnerships between emergency assistance and services that address underlying issues contributing to financial distress. Several studies (Babbington \& DonatoHunt, 2007; Engels, Nissim \& Landvogt, 2009; Frederick \& Goddard, 2008; Gribble, 2006; King et. al. 2009; Landvogt, 2006; Nguyen, 2011) have acknowledged a need in the emergency assistance sector for more holistic approaches, and this study has demonstrated that this approach echoes clients' needs to have a holistic account of their personal circumstances heard and responded to. The response could be undertaken via two means, the first being building stronger partnerships with programs and services across agencies, as well as training and encouraging emergency assistance workers to actively conduct referrals and link clients into these resources (Barker, 2004; Gribble, 2006; Nguyen, 2011). The second option is for increased inter agency network and cross referral service development (Barker, 2004; FaHCSIA, 2012; Gribble, 2006; Nguyen, 2011).

Implication : Reviewing the types of emergency assistance provided, including the consideration of a combination of vouchers and healthy food parcels

The final implication of this study suggest the need for review of the types of emergency assistance provided. Whilst the limitations faced by agencies in providing emergency assistance are significant, it was evident from participant accounts that an inability to get suitable food was disempowering. Although vouchers offer choice, concerns are noted with these, including reselling for less than face value and spending on items for which the voucher was not intended (ACOSS, 2011a). However, restricting vouchers on the basis of those factors positions agencies as paternalistic and subjective arbiters, which is disempowering to emergency assistance clients. It is recommended that a combination of vouchers and food be explored, allowing choice and thus the development of a sense of capacity and dignity whilst also providing basic staples for complete meals. This implication 
not only reflects clients' needs as identified in this current, and previous studies, but also may alleviate agency concerns associated with solely supplying vouchers.

\section{Limitations}

This study does not represent all emergency assistance clients. It has aimed to draw attention to and explore the stories and experiences of a small section of this population in South Australia This small sample size and qualitative approach used to elicit perspectives limits the ability to generalize study findings, however the approach used is approved for the purposes of exploration and highlighting the complex experiences of a sample of emergency assistance recipients in South Australia..

\section{Conclusion}

The three implications that emerge from this study mirror the themes drawn from interviews with participants around the importance of having their humanity recognised. They reflect the centrality of empathy and respect for the whole person, and the importance of interpersonal skills to emergency assistance provision. The themes and implications recognise the complex and embedded nature of personal circumstances and the need to empower and build capacity accordingly. In short, this study, as the first to specifically examine the South Australian context contributes to the ongoing development of literature exploring what should constitute 'best practice' for emergency assistance services. It also enrichens existing work, such as that by Frederick and Goddard (2008), demonstrating not only reoccurring and reinforcing concepts but also, through a range of contrasting findings, the diverse and complex experiences of emergency assistance clients 


\section{References}

Anglicare Australia (2010). Reaching the limits: A profile of emergency relief provision across Anglicare Australia agencies, Canberra, Anglicare Australia.

AnglicareSA (2014). Turn Away Census November 2014, AnglicareSA, South Australia.

Anglicare Victoria (2003). Discussion paper: Emergency relief program Anglicare Victoria's response, Victoria, Anglicare Victoria.

Australian Council of Social Services (ACOSS) (1997a). People in financial crisis: National emergency relief data collection project, ACOSS Paper 93, New South Wales, ACOSS Paper 91, New South Wales, ACOSS.

Australian Council of Social Services (ACOSS) (1997b). People in financial crisis II: National emergency relief data collection project, ACOSS Paper 93, New South Wales, ACOSS.

Australian Council of Social Services (ACOSS) (1999). People in financial crisis III: National emergency relief data collection project, ACOSS Paper 101, New South Wales, ACOSS.

Australian Council of Social Services (ACOSS) (2008). Who is missing out: Hardship among low income Australians, ACOSS Information Paper, New South Wales, ACOSS.

Australian Council of Social Services (ACOSS) (2011a). Emergency Relief Handbook, $4^{\text {th }}$ edn, New South Wales, ACOSS.

Australian Council of Social Services (ACOSS) (2011b). Australian community sector survey, ACOSS Paper 173, New South Wales, ACOSS.

Australian Council of Social Services (ACOSS) (2011c). Poverty Report: October 2011 update, New South Wales, ACOSS.

Babbington, S. \& Donato-Hunt, C. (2007). When there isn't enough to eat: The food insecurity of Anglicare Sydney's emergency relief clients Wollongong, New South Wales, Anglican Diocese of Sydney.

Barker, T. (2004). Local Networks: How and why, paper presented at the Heads, hearts and hands-on emergency relief conference, 25-26 March, 30-34, Victoria, Victorian Council of Social Services.

Bath, B. (1985). 'Emergency relief: more funds not the answer', Australian Social Welfare: Impact, 15(3), 27-31.

Braun, V. \& Clarke, V. (2006). 'Using thematic analysis in psychology', Qualitative Research in Psychology, 3 (2), 77-101.

Engels, B. (2006). The provision of emergency relief and contemporary pressures for change in metropolitan Melbourne, Victoria, RMIT University \& Emergency Relief Victoria Network.

Engels, B. Nissim, R. \& Landvogt, K. (2009). Under pressure: Costs of living, financial hardship and emergency relief in Victoria, Victoria, Victorian Council of Social Services \& Emergency Relief Victoria Network.

Commonwealth Department of Families, Housing, Community Services and Indigenous Affairs (FaHCSIA) (2012). 'Financial Management Program Guidelines: Version 1.6', Canberra, FaHCSIA.

Frederick, J. \& Goddard, C. (2008). 'Sweet and sour charity: Experiences of receiving emergency relief in Australia', Australian Social Work, 61 (3) 269-284.

Green, V. Zappala, G. \& Parker, B (2000). Factors associated with financial disadvantage: a case study of The Smith Family's Emergency Help program in NSW, Research and Advocacy Briefing Paper No. 5, Sydney, The Smith Family.

Gribble, S. (2006). Talking to each other: An analysis of networking in the Victorian emergency relief sector, Melbourne, Victorian Council of Social Services.

Griffiths, D. (1975). Emergency relief: a report prepared for the Social Welfare Commission, Canberra, Social Welfare Commission. 
King, S. Bellamy, J. Swann, N. Gavarotto, R. \& Coller, P. (2009). Social exclusion the Sydney experience, Policy Unit Research Paper, Sydney, Anglican Diocese of Sydney.

Landvogt, K. (2006). 'The 'big idea' of emergency relief,' Just Policy, 40, 54-57.

Liddell, M. (2000). The management of emergency relief: Issues and options, A report for Frankston Community Support and Information Centre. Melbourne, Department of Social Work and Human Services \& Monash University.

Mitchell, M. (2004). The rights perspective, paper presented at the Heads, hearts and handson emergency relief conference, 25-26 March, 47-50, Victoria, Victorian Council of Social Services.

National Council of Social Services (NCOSS) (1985). 'Emergency relief: the current crisis', Australian Social Welfare: Impact, 15(5-6), 38-39.

Neuman, W. (2000(). Social research methods: Qualitative \& quantitative approaches, $4^{\text {th }}$ edn, USA, Allyn \& Bacon.

Nguyen, M.T.T (2011). Ending the stopgap: Casework in emergency relief services, Melbourne, Community Information Victoria \& Cranbourne Information and Support.

Poppendieck, J. (1998). Sweet charity? Emergency food and the end of entitlement, New York: Viking.

Sarantakos, S. (1998). Social research, $2^{\text {nd }}$ edn, Melbourne, Macmillan Education.

Smith, C. (2004). The value of peak bodies, paper presented at the Heads, hearts and hands-on emergency relief conference, 25-26 March, 51-53, Victoria, Victorian Council of Social Services.

South Australian Council of Social Services (SACOSS) (2011). At the crossroads: Financial counselling in South Australia, SACOSS Information Paper, South Australia, SACOSS.

Wilson, A. (2006). Emergency relief Victoria survey report, Melbourne, Emergency Relief Victoria. 


\section{Biographical notes}

Tanya Mackay is a qualified social worker and a PhD candidate at the University of South Australia. Additionally, she has undertaken work in a research assistant capacity at the Australian Centre for Community Services Research at Flinders University.

Ian Goodwin-Smith (PhD) is the Director of the Australian Centre for Community Services Research at Flinders University. He has nested research interests in social theory, social policy and social services, all of which exist within an overall concern for social justice and social inclusion. His work spans theoretical and empirical investigations, and is always attached to an inclusive political purpose. 